In 2015 (January to December), the average time for primary review for all original manuscripts submitted to Circulation Journal was 10.0 days.

\title{
Message From the Editor-in-Chief
}

Editorial Statistics and Best Reviewers Award for 2015

Hiroaki Shimokawa

\section{Reviews}

The Who, What, Why, When, How and Where of Vasospastic Angina

John F. Beltrame, Filippo Crea, Juan Carlos Kaski, Hisao Ogawa, Peter Ong, Udo Sechtem, Hiroaki Shimokawa, C. Noel Bairey Merz on behalf of the Coronary Vasomotion Disorders International Study Group (COVADIS)

Role of Coronary Microvascular Dysfunction in Takotsubo Cardiomyopathy

Cristiana Vitale, Giuseppe MC Rosano, Juan Carlos Kaski

Pearls and Pitfalls in Catheter Ablation of Persistent Atrial Fibrillation

Li-Wei Lo, Yenn-Jiang Lin, Shih-Lin Chang, Yu-Feng Hu, Fa-Po Chung, Shih-Ann Chen

\section{Editorials}

Taking Control of the Time Bomb in Abdominal Aortic Aneurysm Hiroki Aoki

Triple Antithrombotic Therapy - Always One Too Many? Hidehira Fukaya, Junya Ako

How Should We Treat Myocardial Infarction Patients With Multivessel Disease? - Staged Revascularization? Or Stent Them All Acutely? -

Kenichi Tsujita, Kenshi Yamanaga, Koichi Kaikita, Seiji Hokimoto, Hisao Ogawa

Eicosapentaenoic Acid Added to Strong Statin Therapy

Shiro Nakahara, Takaaki Komatsu, Isao Taguchi

Low-Density Lipoprotein Cholesterol Level and Statin Therapy in Patients With Acute Myocardial Infarction (Cholesterol Paradox)

Tsuyoshi Nozue

Ischemic Burden Size, Left Ventricular Function or Both for Better Revascularization Outcomes? Tomoaki Nakata, Koki Abe

Pentraxin 3 - A Link Between Obesity, Inflammation and Vascular Disease? Francesco Violi, Daniele Pastori

Lipoprotein(a): Revisiting a Next Therapeutic Target Kouji Kajinami 


\section{Original Articles}

\section{Aortic Disease}

Inverse Correlation Between Calcium Accumulation and the Expansion Rate of Abdominal Aortic Aneurysms

Atsuko Nakayama, Hiroyuki Morita, Naoto Hayashi, Yukihiro Nomura, Katsuyuki Hoshina, Kunihiro Shigematsu, Hiroshi Ohtsu, Tetsuro Miyata, Issei Komuro

\section{Arrhythmia/Electrophysiology}

Pronounced Shortening of QT Interval With Mexiletine Infusion Test in Patients With Type 3 Congenital Long QT Syndrome

Moritoshi Funasako, Takeshi Aiba, Kohei Ishibashi, Ikutaro Nakajima, Koji Miyamoto, Yuko Inoue, Hideo Okamura, Takashi Noda, Shiro Kamakura, Toshihisa Anzai, Teruo Noguchi, Satoshi Yasuda, Yoshihiro Miyamoto, Kengo Fukushima Kusano, Hisao Ogawa, Wataru Shimizu

Early Recurrence After Pulmonary Vein Isolation of Paroxysmal Atrial Fibrillation With Different Ablation Technologies - Prospective Comparison of Radiofrequency vs. Second-Generation Cryoballoon Ablation -

Shinsuke Miyazaki, Akio Kuroi, Hitoshi Hachiya, Hiroaki Nakamura, Hiroshi Taniguchi, Noboru Ichihara, Takamitsu Takagi, Jin Iwasawa, Yoshito lesaka

Effects of Triple Therapy in Patients With Non-Valvular Atrial Fibrillation Undergoing Percutaneous Coronary Intervention Regarding Thromboembolic Risk Stratification

Antonia Sambola, Maria Mutuberría, Bruno García del Blanco, Albert Alonso, José A. Barrabés, Fernando Alfonso, Héctor Bueno, Angel Cequier, Javier Zueco, Oriol Rodríguez-Leor, Eduard Bosch, Pilar Tornos, David García-Dorado

\section{Cardiovascular Intervention}

Clinical Outcomes of Drug-Eluting Stents vs. Bare-Metal Stents in Acute Myocardial Infarction Patients Under Dialysis - A Nationwide Cohort Study -

Dong-Yi Chen, Chun-Tai Mao, Ming-Lung Tsai, Ming-Jer Hsieh, Yu-Sheng Lin, Wen-Jin Cherng, MingShien Wen, Chao-Hung Wang, I-Chang Hsieh, Ming-Jui Hung, Chun-Chi Chen, Tien-Hsing Chen

Culprit Vessel-Only vs. Staged Multivessel Percutaneous Coronary Intervention Strategies in Patients With Multivessel Coronary Artery Disease Undergoing Primary Percutaneous Coronary Intervention for ST-Segment Elevation Myocardial Infarction

Toshiaki Toyota, Hiroki Shiomi, Tomohiko Taniguchi, Takeshi Morimoto, Yutaka Furukawa, Yoshihisa Nakagawa, Minoru Horie, Takeshi Kimura on behalf of the CREDO-Kyoto AMI Registry Investigators

The Leipzig Prospective Drug-Eluting Balloon-Registry - Outcome of 484 Consecutive Patients Treated for Coronary In-Stent Restenosis and De Novo Lesions Using Paclitaxel-Coated Balloons -

Madlen Uhlemann, Sven Möbius-Winkler, Jennifer Adam, Sandra Erbs, Norman Mangner, Marcus Sandri, Enno Boudriot, Michael Woinke, Gerhard C. Schuler, Axel Linke

\section{Cardiovascular Surgery}

Novel Scoring System to Predict Ineligibility for Bridge to Implantable Left Ventricular Assist Device as Destination Therapy Before Extracorporeal Ventricular Assist Device Implantation - For the Coming Era of Destination Therapy in Japan -

Daisuke Nitta, Koichiro Kinugawa, Teruhiko Imamura, Miyoko Endo, Toshiro Inaba, Hisataka Maki, Eisuke Amiya, Masaru Hatano, Osamu Kinoshita, Kan Nawata, Shunei Kyo, Minoru Ono

Risk Stratification for Cardiac Allograft Vasculopathy in Heart Transplant Recipients - Annual Intravascular Ultrasound Evaluation -

Takuma Sato, Osamu Seguchi, Hatsue Ishibashi-Ueda, Masanobu Yanase, Norihiro Okada, Kensuke Kuroda, Eriko Hisamatsu, Haruki Sunami, Takuya Watanabe, Seiko Nakajima, Kyoichi Wada, Hiroki Hata, Tomoyuki Fujita, Norihide Fukushima, Junjiro Kobayashi, Takeshi Nakatani

\section{Heart Failure}

Determinants and Prognostic Impact of Hyperuricemia in Hospitalization for Acute Heart Failure Wei-Ming Huang, Pai-Feng Hsu, Hao-Min Cheng, Dai-Yin Lu, Yu-Lun Cheng, Chao-Yu Guo, Shih-Hsien Sung, Wen-Chung Yu, Chen-Huan Chen 
Low-Normal Serum Sodium and Heart Failure-Related Events in Patients With Heart Failure With Preserved Left Ventricular Ejection Fraction

Hiroaki Kusaka, Seigo Sugiyama, Eiichiro Yamamoto, Eiichi Akiyama, Yasushi Matsuzawa, Yoshihiro Hirata, Koichiro Fujisue, Hirofumi Kurokawa, Junichi Matsubara, Koichi Sugamura, Hirofumi Maeda, Hideaki Jinnouchi, Kunihiko Matsui, Hisao Ogawa

Predictors of Worsening Renal Function in Patients With Acute Decompensated Heart Failure Treated by Low-Dose Carperitide Yuichi Kawase, Kazushige Kadota, Takeshi Tada, Reo Hata, Keiichiro Iwasaki, Takeshi Maruo, Harumi Katoh, Kazuaki Mitsudo

\section{Hypertension and Circulatory Control}

Effect of Diuretic or Calcium-Channel Blocker Plus Angiotensin-Receptor Blocker on Diastolic Function in Hypertensive Patients

Norihisa Toh, Katsuhisa Ishii, Hajime Kihara, Katsuomi Iwakura, Hiroyuki Watanabe, Junichi Yoshikawa, Hiroshi Ito for Effect of ARB/Diuretics on Diastolic Function in Patients with Hypertension 2 (EDEN2) trial investigators

\section{Imaging}

Prognostic Value of Cardiac Sympathetic Nerve Imaging Using Long-Term Follow-up Data - Ischemic

vs. Non-Ischemic Heart Failure Etiology -

Shinro Matsuo, Kenichi Nakajima, Tomoaki Nakata

\section{Ischemic Heart Disease}

Factors Affecting Platelet Reactivity 2 Hours After P2Y12 Receptor Antagonist Loading in Primary Percutaneous Coronary Intervention for ST-Elevation Myocardial Infarction - Impact of Pain-toLoading Time -

Ioanna Xanthopoulou, Periklis Davlouros, Grigorios Tsigkas, Nikolaos Koutsogiannis, Sotirios Patsilinakos, Spyridon Deftereos, George Hahalis, Dimitrios Alexopoulos

Effects of the Addition of Eicosapentaenoic Acid to Strong Statin Therapy on Inflammatory Cytokines and Coronary Plaque Components Assessed by Integrated Backscatter Intravascular Ultrasound Toshiyuki Niki, Tetsuzo Wakatsuki, Koji Yamaguchi, Yoshio Taketani, Hiroyasu Oeduka, Kenya Kusunose, Takayuki Ise, Takashi Iwase, Hirotsugu Yamada, Takeshi Soeki, Masataka Sata

Effect of Statin Treatment and Low-Density Lipoprotein-Cholesterol on Short-Term Mortality in Acute Myocardial Infarction Patients Undergoing Primary Percutaneous Coronary Intervention - Multicenter Registry From Tokyo CCU Network Database -

Mizuki Miura, Masao Yamasaki, Yukari Uemura, Masatomo Yoshikawa, Katsumi Miyauchi, Hiroyuki Tanaka, Hideki Miyachi, Jun Yamashita, Makoto Suzuki, Takeshi Yamamoto, Ken Nagao, Issei Komuro, Morimasa Takayama

Plasma Glucagon-Like Peptide-1 and Tissue Characteristics of Coronary Plaque in Non-Diabetic Acute Coronary Syndrome Patients

Takayuki Mitsuhashi, Kiyoshi Hibi, Masaaki Konishi, Nobuhiko Maejima, Noriaki Iwahashi, Kengo Tsukahara, Masami Kosuge, Toshiaki Ebina, Satoshi Umemura, Kazuo Kimura

Intravascular Ultrasound Guidance vs. Angiographic Guidance in Primary Percutaneous Coronary Intervention for ST-Segment Elevation Myocardial Infarction - Long-Term Clinical Outcomes From the CREDO-Kyoto AMI Registry -

Kenji Nakatsuma, Hiroki Shiomi, Takeshi Morimoto, Kenji Ando, Kazushige Kadota, Hiroki Watanabe, Tomohiko Taniguchi, Takashi Yamamoto, Yutaka Furukawa, Yoshihisa Nakagawa, Minoru Horie, Takeshi Kimura on behalf of the CREDO-Kyoto AMI investigators

Long-Term Survival Benefit of Coronary Revascularization in Patients Undergoing Stress Myocardial Perfusion Imaging

Mario Petretta, Wanda Acampa, Stefania Daniele, Emilia Zampella, Roberta Assante, Carmela Nappi, Marco Salvatore, Alberto Cuocolo

Additive Value of Heart Rate Variability in Predicting Obstructive Coronary Artery Disease Beyond Framingham Risk

Hsin-Ru Li, Tse-Min Lu, Hao-Min Cheng, Dai-Yin Lu, Chuen-Wang Chiou, Shao-Yuan Chuang, Albert C. Yang, Shih-Hsien Sung, Wen-Chung Yu, Chen-Huan Chen 


\section{Metabolic Disorder}

Pentraxin 3 and Platelet Activation in Obese Patients After Gastric Banding

Francesca Santilli, Maria Teresa Guagnano, Paolo Innocenti, Liberato Aceto, Natale Vazzana, Stefano Lattanzio, Rossella Liani, Romina Tripaldi, Valeria Creato, Mario Romano, Giovanni Davi

Lipoprotein(a) in Familial Hypercholesterolemia With Proprotein Convertase Subtilisin/Kexin Type 9 (PCSK9) Gain-of-Function Mutations

Hayato Tada, Masa-aki Kawashiri, Taiji Yoshida, Ryota Teramoto, Atsushi Nohara, Tetsuo Konno, Akihiro Inazu, Hiroshi Mabuchi, Masakazu Yamagishi, Kenshi Hayashi

\section{Valvular Heart Disease}

Significance of Coronary Artery Disease and Left Ventricular Afterload in Unoperated Asymptomatic Aortic Stenosis

Kentaro Shibayama, Masao Daimon, Hiroyuki Watanabe, Takayuki Kawata, Sakiko Miyazaki, Ryoko Morimoto-Ichikawa, Masaki Maruyama, Shuo-Ju Chiang, Katsumi Miyauchi, Hiroyuki Daida

Prognostic Significance of ST-Segment Elevation in Leads $\mathrm{V}_{1-2}$ in Patients With Severe Aortic Stenosis Tomohiko Taniguchi, Hiroki Shiomi, Masami Kosuge, Takeshi Morimoto, Kenji Nakatsuma, Masataka Nishiga,

Tomoki Sasa, Naritatsu Saito, Takeshi Kimura

\section{Images in Cardiovascular Medicine}

Coronary Stenosis as an Innocent Bystander in Acute Coronary Syndrome Italo Porto, Domenico D'Amario, Lazzaro Paraggio, Filippo Crea

Cardiac Arrest Triggered by Subepicardial Aneurysm Without Cardiac Rupture Eriko Hasumi, Katsuhito Fujiu, Teruhiko Imamura, Hiroshi Iwata, Daigo Sawaki, Yumiko Hosoya, Jiro Ando, Toshiya Kojima, Yu Shimizu, Gaku Oguri, Takumi Matsubara, Masaru Hatano, Hiroshi Akazawa, Masafumi Watanabe, Minoru Ono, Issei Komuro

\section{Letters to the Editor}

Does Age Have an Effect on Systemic Inflammatory Response?

(Letter) Orhan Gokalp, Ali Gurbuz, Nihan Karakas Yesilkaya, Yuksel Besir, Gamze Gokalp

(Reply) Suguru Ohira, Hitoshi Yaku

Screening of Coronary Artery Disease in Diabetic Patients: Who and How?

(Letter) Jesus M de la Hera, Maria Martín, Cecilia Corros, Ana García-Campos, Juan Calvo

(Reply) Michio Shimabukuro, Saito Taro, Hiroaki Masuzaki, Masataka Sata 